\title{
INTERFERENCE ALIGNMENT USING REWEIGHTED NUCLEAR NORM MINIMIZATION
}

\author{
Gokul Sridharan and Wei Yu \\ Edward S. Rogers Department of Electrical and Computer Engineering \\ University of Toronto, Canada \\ Email: gsridharan@comm.utoronto.ca,weiyu@comm.utoronto.ca
}

\begin{abstract}
This paper proposes an algorithm to compute the transmit beamformers for linear interference alignment for the MIMO interference channel and the MIMO interfering multipleaccess/broadcast channel without symbol extensions. We first formulate the interference alignment problem as a rank minimization problem with linear constraints, then approximate the matrix rank by the nuclear norm. We further propose the use of an iterative reweighted nuclear norm approach and show that adaptive reweighting can significantly improve the algorithm's ability to find aligned beamformers. Simulation results show that the proposed algorithm is able to provide more interference-free dimensions and also converges faster than a previously proposed rank-constrained rank-minimization approach for interference alignment.
\end{abstract}

Index Terms- Interference alignment, interference mitigation, rank minimization, nuclear norm minimization, reweighting.

\section{INTRODUCTION}

Since the landmark development of interference alignment in $[1,2]$, there have been significant interests in applying alignment techniques for various practical networks such as the MIMO cellular networks [3]. While the original work on interference alignment was based on asymptotic symbol extension, alignment schemes where only finite extensions over time or frequency are allowed are of practical interest. With the current trend in cellular networks to move towards smaller cells and to enable cooperation among multiple base-stations, managing interference through linear interference alignment holds significant promise.

While it is straightforward to compute the transmit and receive beamformers for interference alignment when closed form expressions are available (e.g. the 3-user $N \times N$ interference channel [1] and the 2-cell broadcast channel [3]), iterative algorithms are typically used when such solutions are not readily available [4-8]. In [4], an iterative algorithm for the MIMO interference channel based on minimizing the sum of interference power at all the receivers was proposed. In [6], the algorithm of [4] is extended to the MIMO interfer- ing cellular networks. While algorithms of $[4,6]$ are known to converge, they typically need several thousand iterations.

This paper is based on a rank minimization approach to finding linear beamformers for linear interference alignment, similar to the approach taken in [7]. In [7] the problem of finding beamformers for interference alignment was posed as a matrix rank minimization problem subject to rank constraints. The algorithm of [7] involves iteratively solving two semidefinite programs with the objective of minimizing the nuclear norm of a matrix. This algorithm was shown to be better than the algorithm of [4] for systems with time/frequency extensions.

In [7], the authors further show that the algorithm proposed in [4] is equivalent to minimizing the Frobenius norm (matrix analogue of the $\ell_{2}$-norm for vectors) of the same matrix. Since minimizing the $\ell_{2}$-norm or the Frobenius norm is known to result in many small singular values as opposed to few non-zero singular values, this provides a plausible explanation for the slow convergence of algorithms presented in $[4,6]$. While the nuclear norm minimization approach taken in [7] is better suited for inducing sparsity, the impact of the convex constraint that is used in lieu of the rank constraint on the optimization problem is difficult to assess. Further, nuclear norm has known drawbacks in inducing sparsity [9] and better approximations to rank are needed to generate the maximum possible interference-free dimensions.

In this work, we focus on linear interference alignment without symbol extensions for generic channels and formulate the problem of finding aligned beamformers as a rank minimization problem subject to linear constraints. While the formulation is similar in spirit to [7], there are significant differences. First, we only optimize over transmit beamformers to align interference at the receivers; the receive beamformers are chosen subsequently to zero-out the aligned interference. This approach does not require alternating between the forward and backward channels and reduces the total number of variables to optimize over. Second, we avoid imposing rank constraints on the transmit beamformers by enforcing the transmit beamformers to be in reduced row echelon form, where all superfluous variables subject to the rank constraint are eliminated. Third, rather than approximating the rank of a matrix by its nuclear norm, we use an iterative approach 
where rank is approximated using a series of weighted nuclear norms. This approach is motivated by recent works in matrix rank minimization $[9,10]$ that suggest that the nuclear norm might not be as effective in inducing sparsity as previously thought and that an iterative reweighting procedure [10] can significantly improve upon the solution provided by direct nuclear norm minimization. Throughout this paper, we make significant use of these reweighting heuristics developed for matrix rank minimization [11-14].

\section{SYSTEM MODEL}

We consider a $K$-user MIMO interference channel with $M_{t}$ transmit antennas at each transmitter and $M_{r}$ receive antennas at each receiver. Each transmitter is assumed to be sending $d$ data streams to its receiver. The channel from the $i^{t h}$ transmitter to the $j^{t h}$ receiver is denoted by the $M_{r} \times M_{t}$ matrix $\mathbf{H}_{i j}$. The channel matrices are assumed to be generic. Assuming perfect synchronization at all the transmitters, the received signal at the $i^{\text {th }}$ receiver after appropriate sampling is given by

$$
\mathbf{y}_{i}=\sum_{j=1}^{K} \mathbf{H}_{j i} \mathbf{V}_{j} \mathbf{s}_{j}+\mathbf{n}_{i}
$$

where $\mathbf{s}_{j}$ is the $d \times 1$ symbol vector transmitted by the $j^{\text {th }}$ transmitter, $\mathbf{V}_{j}$ is the $M_{t} \times d$ linear precoding matrix, $\mathbf{y}_{i}$ is the $M_{r} \times 1$ received signal vector at the $i^{t h}$ receiver, and $\mathbf{n}_{i}$ is the additive white Gaussian noise vector with covariance $\sigma^{2} \mathbf{I}$.

The received signal at the $i^{\text {th }}$ receiver is processed using a linear zero-forcing filter denoted by the $M_{r} \times d$ matrix $\mathbf{U}_{i}$. After linear processing at the receiver, the signal can be written as

$$
\mathbf{U}_{i}^{H} \mathbf{y}_{i}=\mathbf{U}_{i}^{H} \mathbf{H}_{i i} \mathbf{V}_{i} \mathbf{s}_{i}+\sum_{j=1, j \neq i}^{K} \mathbf{U}_{i}^{H} \mathbf{H}_{j i} \mathbf{V}_{j} \mathbf{s}_{j}+\mathbf{U}_{i}^{H} \mathbf{n}_{i}
$$

We denote the space occupied by interference at the $i^{\text {th }}$ receiver as the range of the following matrix

$$
\begin{gathered}
\mathbf{R}_{i}=\left[\mathbf{H}_{1 i} \mathbf{V}_{1}, \ldots, \mathbf{H}_{(i-1) i} \mathbf{V}_{i-1}, \mathbf{H}_{(i+1) i} \mathbf{V}_{i+1},\right. \\
\left.\ldots, \mathbf{H}_{K i} \mathbf{V}_{K}\right]
\end{gathered}
$$

\section{RANK MINIMIZATION APPROACH}

For the system described above, the conditions for linear interference alignment when symbol extensions over time or frequency are not allowed can be stated as follows:

$$
\begin{aligned}
\mathbf{U}_{i}^{H} \mathbf{H}_{j i} \mathbf{V}_{j} & =\mathbf{0} \forall i \neq j, \\
\operatorname{rank}\left(\mathbf{U}_{i}^{H} \mathbf{H}_{i i} \mathbf{V}_{i}\right) & =d \forall i .
\end{aligned}
$$

For a given system, it is not always possible to satisfy the conditions in (4) and (5). A preliminary check on feasibility is to make sure that the given system is proper [15]. A $K$ user $M_{t} \times M_{r}$ interference channel is said to be proper if $\left(M_{r}+M_{t}\right) /(K+1) \leq d$. While not all proper systems are feasible [16], improper systems have been shown to be almost surely infeasible [17]. In this paper, we only consider proper systems that are known to be feasible.

Assuming channels to be generic allows us to restate the conditions in (4) and (5) in a manner that directly leads to the rank minimization approach. Since direct channels do not play a role in (4), the condition in (5) is automatically satisfied whenever $\mathbf{U}_{i}$ and $\mathbf{V}_{i}$ have rank $d$ and whenever the channels are generic [15]. As a further consequence of channels being generic, satisfying (4) is equivalent to the condition that the set of transmit precoders $\left\{\mathbf{V}_{i}\right\}_{i=1}^{K}$ (henceforth denoted as $\mathbf{V}$ ) is such that there are at least $d$ interferencefree dimensions at each receiver before any linear processing. In essence, generic channels ensure that the intersection between useful signal subspace $\left(\operatorname{span}\left(\mathbf{H}_{i i} \mathbf{V}_{i}\right)\right.$ ) and interference subspace $\left(\operatorname{span}\left(\mathbf{R}_{i}\right)\right)$ is almost surely zero dimensional, provided that the $\operatorname{rank}\left(\mathbf{R}_{i}\right) \leq\left(M_{r}-d\right) \forall i$. Thus the requirements for interference alignment can be alternately stated as

$$
\begin{array}{r}
\operatorname{rank}\left(\mathbf{R}_{i}\right) \leq M_{r}-d \forall i, \\
\operatorname{rank}\left(\mathbf{V}_{i}\right)=d \forall i .
\end{array}
$$

Given a set of transmit precoders $\mathbf{V}$ that satisfy the above conditions, designing the receive filters is straightforward.

Using (6) and (7) we reframe the requirements for interference alignment as an optimization problem to minimize the rank of a matrix. First, we note that the condition (7) is immediately satisfied by restricting $\mathbf{V}_{i}^{T}$ to be of the row reduced echelon form $\left[\mathbf{I}_{d \times d} \tilde{\mathbf{V}}_{i}^{T}\right]$. Note that there is no loss of generality in requiring $\mathbf{V}_{i}$ to be of this form as any set of $d$ linearly independent vectors can be written in this form through column transformations. Thus, the interference alignment conditions can be posed as the following rank minimization problem subject to linear constraints:

$$
\begin{aligned}
& \operatorname{minimize} \sum_{i=1}^{K} \operatorname{rank}\left(\mathbf{R}_{i}\right) \\
& \text { subject to } \mathcal{A}_{i}\left(\mathbf{V}_{i}\right)=\mathbf{b}_{i}, \mathcal{C}_{i}(\mathbf{V})=\operatorname{vec}\left(\mathbf{R}_{i}\right) \forall i,
\end{aligned}
$$

where $\mathcal{A}_{i}\left(\mathbf{V}_{i}\right)=\mathbf{b}_{i}$ represents the collection of all linear constraints that need to be satisfied by the transmit precoders, e.g. $\mathbf{V}_{i}(1: d, 1: d)=\mathbf{I}_{d \times d}$, and $\mathcal{C}_{i}(\mathbf{V})=\operatorname{vec}\left(\mathbf{R}_{i}\right)$ represents the relationship between the entries in $\mathbf{R}_{i}$ and the transmit precoders, as given in (3). This is a rank minimization problem subject to linear constraints and differs from the rank minimization subject to rank constraint formulation presented in [7] in two key aspects. The formulation presented in [7] is as given below:

$$
\operatorname{minimize} \sum_{i=1}^{K} \operatorname{rank}\left(\mathbf{U}_{i}^{H} \mathbf{R}_{i}\right)
$$

subject to $\lambda_{\min }\left(\mathbf{U}_{i}^{H} \mathbf{H}_{i i} \mathbf{V}_{i}\right) \geq \epsilon, \mathcal{C}_{i}(\mathbf{V})=\operatorname{vec}\left(\mathbf{R}_{i}\right) \forall i$, 
where $\epsilon>0$. The constraint on the minimum eigenvalue of the received signal space $\mathbf{U}_{i}^{H} \mathbf{H}_{i i} \mathbf{V}_{i}$ ensures that (a) all transmit and receive beamformer matrices have rank $d$ and (b) the received signal space $\mathbf{U}_{i}^{H} \mathbf{H}_{i i} \mathbf{V}_{i}$ is not rank deficient. In our formulation, while (a) is addressed by imposing the row reduced echelon form on $\mathbf{V}_{i}^{T}$, (b) is assured by assuming channels to be generic, allowing us to only impose linear equality constraints in our formulation. Further, for any choice of $\epsilon>0$, the constraint on the smallest eigenvalue places a restriction on the received signal space and the optimization is no longer a pure pursuit to align interference. While this is a necessary constraint when channels are not generic, this constraint can be dropped for generic channels. Since direct channels and the received signal space play no part in (8), this formulation is not hindered by any constraints on the received signal space. Finally, the proposed methodology to solve (9) approximates rank using the nuclear norm and involves alternating between two semidefinite programs, one to optimize the receive beamformers and the other to optimize the transmit beamformers. Since we only optimize the transmit beamformers, our approach involves iteratively solving only one set of semidefinite programs.

\section{REWEIGHTED NUCLEAR NORM MINIMIZATION}

The rank minimization formulation in (8) has been extensively studied in parallel in the investigations of $\ell_{1}$-norm minimization problem for compressed sensing [11-13]. Typically, rank is approximated using the nuclear norm of the matrix, which is the matrix counterpart of $\ell_{1}$-norm for vectors. Nuclear norm is the sum of the singular values of a matrix and is known to be the convex envelope of rank [18]. Using this approximation for rank, the rank minimization problem (8) can be posed as:

$$
\begin{aligned}
& \operatorname{minimize}\|\mathbf{R}\|_{*} \\
& \text { subject to } \mathcal{A}_{i}\left(\mathbf{V}_{i}\right)=\mathbf{b}_{i}, \mathcal{C}_{i}(\mathbf{V})=\operatorname{vec}\left(\mathbf{R}_{i}\right) \forall i,
\end{aligned}
$$

where we denote the block diagonal matrix consisting of the matrices $\left\{\mathbf{R}_{i}\right\}_{i=1}^{K}$ as $\mathbf{R}$. Note that $\sum_{i=1}^{K}\left\|\left(\mathbf{R}_{i}\right)\right\|_{*}=\|\mathbf{R}\|_{*}$.

While nuclear norm is well known for inducing sparsity, further improvement is possible using an iterative reweighting procedure. Such a reweighting procedure was proposed in [9] for $\ell_{1}$-norm minimization and was extended to nuclear norm minimization in [10]. We use the reweighted nuclear norm minimization procedure outlined in [10] to solve (8). This procedure reformulates the rank minimization in (8) as

$$
\begin{aligned}
& \operatorname{minimize}\left\|\mathbf{W}_{1} \mathbf{R} \mathbf{W}_{2}\right\|_{*} \\
& \text { subject to } \mathcal{A}_{i}\left(\mathbf{V}_{i}\right)=\mathbf{b}_{i}, \mathcal{C}_{i}(\mathbf{V})=\operatorname{vec}\left(\mathbf{R}_{i}\right) \forall i,
\end{aligned}
$$

where $\mathbf{W}_{1}$ and $\mathbf{W}_{2}$ are two positive definite matrices that are interpreted to reweight the nuclear norm of $\mathbf{R}$. The iterative procedure involves solving one instance of (11) for fixed
$\mathbf{W}_{1}$ and $\mathbf{W}_{2}$ per iteration, then updating the weights for the next iteration. The choice of the weight update rule affects the overall performance and needs to be chosen carefully. It has been shown in [9] that some of the heuristic weight updates can be interpreted to be equivalent to minimizing a concave surrogate function of rank through a majorizationminimization procedure $[9,10,13]$. In this paper, we consider two weight update rules, one corresponding to minimizing the surrogate function of $\log \operatorname{det}($.$) and the other corre-$ sponding to the surrogate function of $-\operatorname{tr}(\operatorname{inv}()$.$) . The iter-$ ative reweighted nuclear norm minimization to solve (8) can be summarized as

1. Initialize $\mathbf{W}_{1}^{1}=\mathbf{I}, \mathbf{W}_{2}^{1}=\mathbf{I}, \delta$.

2. Iterate over $\mathrm{k}$ :

(a) Solve 11 using weights $\mathbf{W}_{1}^{k}$ and $\mathbf{W}_{2}^{k}$. Let $\mathbf{R}_{\mathrm{opt}}$ be the optimal matrix.

(b) Compute the reduced SVD of $\mathbf{W}_{1}^{k} \mathbf{R}_{\text {opt }} \mathbf{W}_{2}^{k}$, and denote it as $\mathbf{P} \boldsymbol{\Sigma} \mathbf{Q}^{H}$.

(c) Set

$$
\begin{aligned}
& \mathbf{W}_{1}^{k+1}=\left(\left(\mathbf{W}_{1}^{k}\right)^{-1} \mathbf{P} \boldsymbol{\Sigma} \mathbf{P}^{H}\left(\mathbf{W}_{1}^{k}\right)^{-1}+\delta \mathbf{I}\right)^{-\alpha}, \\
& \mathbf{W}_{2}^{k+1}=\left(\left(\mathbf{W}_{2}^{k}\right)^{-1} \mathbf{Q} \boldsymbol{\Sigma} \mathbf{Q}^{H}\left(\mathbf{W}_{2}^{k}\right)^{-1}+\delta \mathbf{I}\right)^{-\alpha} .
\end{aligned}
$$

The parameter $\delta>0$ is a regularization constant to make sure that the weighting matrices are positive definite. When $\alpha=1 / 2$, the update rule corresponds to the $\log \operatorname{det}($.$) ap-$ proximation and when $\alpha=1$, the update rule corresponds to the $-\operatorname{tr}(\operatorname{inv}()$.$) approximation. It is worth noting that the first$ step in this iterative procedure $(k=1)$ is identical to solving (10) and every subsequent step tries to further improve the solution obtained in the previous iteration.

This routine can be shown to converge and at the converged point, we obtain the transmit beamformers $\mathbf{V}_{\text {opt }}$. The receive beamformers at receiver $k$ can then be chosen to be the left-singular vectors corresponding to the $d$ smallest singular values of the matrix $\mathbf{R}_{k}$ computed using $\mathbf{V}_{\text {opt }}$. Since the proposed algorithm only converges to a local optimum, it might not always yield a sufficiently low rank solution so that, even for a proper system known to be feasible, interference cannot be completely nulled out at all the receivers. In such cases, some residual interference leaks into the signal space resulting in fewer degrees of freedom.

Finally, we would like to point out that the formulation in (8) also extends to interfering MIMO multiple-access channel. However, for the interfering MIMO broadcast channel, decomposition of the transmit precoders into the reduced row echelon form is not guaranteed because the transmitted data streams are intended for different users. Hence, for the interfering broadcast channel, we invoke the channel reciprocity argument [4] and solve (8) for the interfering MIMO multiple access channel corresponding to the uplink channel. 


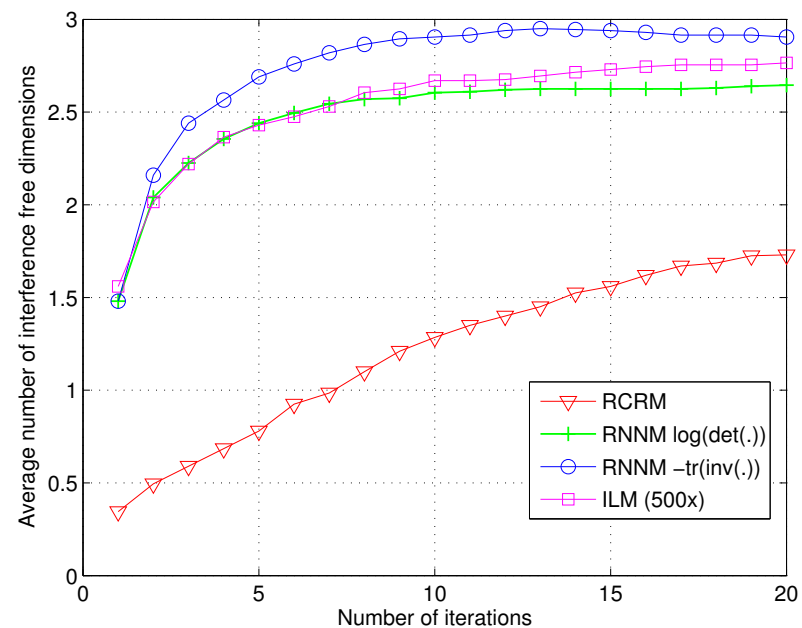

Fig. 1. Interference-free dimensions as a function of iterations for a 4 user $2 \times 3$ interference channel with 1 data stream per user.

\section{SIMULATION RESULTS}

In order to test our algorithm, we consider a 4 -user $2 \times 3$ interference channel with 1 data stream per user - a proper and feasible system [15]. For perfect interference alignment, we need interfering vectors to occupy 2 or fewer dimensions at every receiver. We run reweighted nuclear norm minimization (RNNM) for 20 iterations, with $\delta$ set to 1 . The convex optimization problem in (11) was solved using CVX, a package for specifying and solving convex programs $[19,20]$. We compare our algorithm with the interference leakage minimization (ILM) algorithm [4] and the rank constrained rank minimization algorithm(RCRM) [7] with $\epsilon$ set to $10^{-3}$. An iteration of RCRM involves solving 2 semidefinite programs and one iteration of the algorithm in [4] involves computing 2 eigen decompositions per user. The algorithms are tested over 200 channel realizations with channel coefficients drawn from a real zero-mean unit-variance Gaussian distribution. The algorithms are run for a fixed number of iterations, and the interference-free dimensions at receiver $k$ are counted as the number of singular values of $\mathbf{U}_{k}^{H} \mathbf{H}_{k k} \mathbf{V}_{k}>10^{-3}$ minus the number of singular values of $\mathbf{U}_{k}^{H} \mathbf{R}_{k}>10^{-6}$.

The simulation results are plotted in Fig. 1, where the number of interference-free dimensions for the overall network averaged over all the channel realizations are plotted as a function of the number of iterations. For the ILM algorithm alone, one iteration in the plot corresponds to 500 actual iterations. This scaling is purely for visual purposes and is not to be taken to suggest a matching of complexities between the different algorithms. It is clearly seen that while none of the three algorithms are able to provide the 4 interference-free dimensions possible for such a system, the $-\operatorname{tr}(\operatorname{inv}()$.$) -RNNM$ heuristic is able to provide close to 3 interference-free di- mensions. While the performance of the $-\operatorname{tr}(\operatorname{inv}()$.$) -RNNM$ heuristic is better than the performance of the $\log (\operatorname{det}()$.$) -$ RNNM heuristic, both heuristics perform significantly better than the RCRM algorithm.

We also note that the performance of the RNNM heuristic after the first iteration corresponds to unweighted nuclear norm minimization. The fact that the performance of the RNNM heuristic after the first iteration is better than the RCRM heuristic after one iteration shows that the gain over the RCRM heuristic is not just due to the reweighting of the nuclear norm and that there is a clear advantage of using the proposed rank minimization formulation. The subsequent improvement in the performance of the RNNM heuristic after the first iteration highlights the gains due to the reweighting procedure.

Due to the significant difference in the per iteration complexities, comparison to the ILM algorithm is not straightforward. We can however note that the performance of the ILM algorithm after about 2500 iterations, falls between the performance of the two proposed heuristics (after 10 iterations) with further iterations yielding negligible performance gain.

To understand the better performance of the $-\operatorname{tr}(\operatorname{inv}()$. -RNNM heuristic, we draw parallels to reweighted $\ell_{1}$-norm minimization to provide an intuitive explanation similar to [9]. Rank minimization and nuclear norm minimization of a matrix are equivalent to minimizing the $\ell_{0}$ and $\ell_{1}$ norm of its singular values respectively. While $\ell_{0}$-norm imposes a constant penalty for non-zero singular values, the $\ell_{1}$-norm imposes a linear penalty. While the linear penalty is sparsity inducing, it also unnecessarily incentivizes the reduction of large singular values. The goal of reweighted $\ell_{1}$ norm/nuclear norm minimization is to minimize a weighted sum of singular values such that the large singular values now have small weights. When the weights are chosen to be inversely proportional to the singular values, the resulting RNNM heuristic can be shown to be equivalent to using the $\log \operatorname{det}($.$) surrogate function for rank. Similarly, choos-$ ing weights to be inversely proportional to the square of the singular values can be shown to be equivalent to using the $-\operatorname{tr}(\operatorname{inv}()$.$) surrogate function for rank. This increased$ weighting of very small singular values while suppressing the penalty due large singular values explains the performance difference between the two RNNM heuristics.

\section{CONCLUSION}

In this paper we proposed a rank minimization formulation with linear constraints for linear interference alignment without symbol extensions. The heuristic uses the reweighted nuclear norm minimization framework to solve the resulting optimization problem. Through simulations we established that the proposed algorithm is quick to converge and yields a higher number of interference-free dimensions than the previously proposed rank constrained rank minimization approach. 


\section{REFERENCES}

[1] V. R. Cadambe and S. A. Jafar, "Interferencealignment and degrees of freedom of the K-user interference channel," IEEE Trans. Inf. Theory, vol. 54, no. 8, pp. 3425 -3441 , Aug. 2008.

[2] M. A. Maddah-Ali, A. S. Motahari, and A. K. Khandani, "Communication over MIMO-X channels: Interference alignment, decomposition, and performance analysis," IEEE Trans. Inf. Theory, vol. 54, no. 8, pp. $3457-3470$, Aug. 2008.

[3] C. Suh, M. Ho, and D. N. C. Tse, "Downlink interference alignment," IEEE Trans. Commun., vol. 59, no. 9, pp. 2616-2626, Sep. 2011.

[4] K. Gomadam, V. R. Cadambe, and S. A. Jafar, "A distributed numerical approach to interference alignment and applications to wireless interference networks," IEEE Trans. Inf. Theory, vol. 57, no. 6, pp. 3309-3322, Jun. 2011.

[5] S. W. Peters and R. W. Heath, "Interference alignment via alternating minimization," in Proc. IEEE International Conference on Acoustics, Speech and Signal Processing, Apr. 2009, pp. 2445-2448.

[6] B. Zhuang, R. A. Berry, and M. L. Honig, "Interference alignment in MIMO cellular networks," in Proc. IEEE International Conference on Acoustics, Speech and Signal Processing, May 2011.

[7] D. S. Papailiopoulos and A. G. Dimakis, "Interference alignment as a rank constrained rank minimization," IEEE Trans. Signal Process., vol. 60, no. 8, pp. 42784288, Aug. 2012.

[8] D. A. Schmidt, C. Shi, R. A. Berry, M. L. Honig, and W. Utschick, "Minimum mean squared error interference alignment," in Proc. Asilomar Conference on Signals, Systems and Computers, Nov. 2009, pp. 11061110.

[9] E. J. Candes, M. B. Wakin, and S. P. Boyd, "Enhancing sparsity by reweighted $\ell_{1}$ minimization," Journal of Fourier Analysis and Applications, vol. 14, no. 5, pp. 877-905, 2008.

[10] K. Mohan and M. Fazel, "Reweighted nuclear norm minimization with application to system identification," in Proc. American Control Conference, Jun. 2010, pp. 2953-2959.

[11] B. Recht, M. Fazel, and P. A. Parrilo, "Guaranteed minimum-rank solutions of linear matrix equations via nuclear norm minimization," SIAM Review, vol. 52, no. 3, pp. 471-501, Aug. 2010.
[12] B. Recht, W. Xu, and B. Hassibi, "Necessary and sufficient conditions for success of the nuclear norm heuristic for rank minimization," in Proc. IEEE Conference on Decision and Control, Dec. 2008, pp. 3065-3070.

[13] M. Fazel, H. Hindi, and S. P. Boyd, "Log-det heuristic for matrix rank minimization with applications to Hankel and Euclidean distance matrices," in Proc. American Control Conference, vol. 3, Jun. 2003, pp. 2156-2162.

[14] — , "Rank minimization and applications in system theory," in Proc. American Control Conference, vol. 4, Jun. 2004, pp. 3273-3278.

[15] C. M. Yetis, T. Gou, S. A. Jafar, and A. H. Kayran, "On feasibility of interference alignment in MIMO interference networks," IEEE Trans. Signal Process., vol. 58, no. 9, pp. 4771-4782, Sep. 2010.

[16] C. Wang, T. Gou, and S. A. Jafar, "Subspace alignment chains and the degrees of freedom of the threeuser MIMO interference channel," IEEE Trans. Inf. Theory, submitted for publication. [Online]. Available: http://arxiv.org/abs/1109.4350

[17] M. Razaviyayn, G. Lyubeznik, and Z.-Q. Luo, "On the degrees of freedom achievable through interference alignment in a MIMO interference channel," IEEE Trans. Signal Process., vol. 60, no. 2, pp. $812-821$, Feb. 2012.

[18] M. Fazel, H. Hindi, and S. P. Boyd, "A rank minimization heuristic with application to minimum order system approximation," in Proc. American Control Conference, vol. 6, Jun. 2001, pp. 4734-4739.

[19] CVX Research, Inc., "CVX: Matlab software for disciplined convex programming, version 2.0 beta," September 2012. [Online]. Available: http://cvxr.com/ $\operatorname{cvx}$

[20] M. Grant and S. Boyd, "Graph implementations for nonsmooth convex programs," in Recent Advances in Learning and Control, ser. Lecture Notes in Control and Information Sciences, V. Blondel, S. Boyd, and H. Kimura, Eds. Springer-Verlag Limited, 2008, pp. 95-110. [Online]. Available: http://stanford.edu/ boyd/ graph_dcp.html 